\title{
ON EQUICONTINUITY OF FAMILIES OF MAPPINGS IN A CASE OF VARIABLE DOMAINS
}

\author{
Evgeny Sevost'yanov ${ }^{1}$, Sergei Skvortsov ${ }^{2}$ \\ ${ }^{1}$ Zhytomyr Ivan Franko State University, Zhytomyr, Ukraine; Institute of Applied \\ Mathematics and Mechanics of NAS of Ukraine, Slov'yans'k, Ukraine \\ ${ }^{2}$ Zhytomyr Ivan Franko State University, Zhytomyr, Ukraine \\ esevostyanov2009@gmail.com,serezha.skv@gmail.com
}

Throughout, $D$ and $D^{\prime}$ are domains in $\mathbb{R}^{n}, n \geqslant 2$. In what follows, by $\Gamma(E, F, D)$ we define a family of all paths $\gamma:[a, b] \rightarrow \overline{\mathbb{R}^{n}}: \gamma(a) \in E, \gamma(b) \in F, \gamma(t) \in D$, when $t \in[a, b]$. Given $0<r_{1}<r_{2}<\infty$, denote $A=A\left(x_{0}, r_{1}, r_{2}\right)=\left\{x \in \mathbb{R}^{n}: r_{1}<\left|x-x_{0}\right|<r_{2}\right\}$. Given a family of paths $\Gamma$ in $\mathbb{R}^{n}$, a Borel function $\rho: \mathbb{R}^{n} \rightarrow[0, \infty]$ is called admissible for $\Gamma$, abbr. $\rho \in \operatorname{adm} \Gamma$, if $\int \rho d s \geqslant 1$ for all (locally rectifiable) $\gamma \in \Gamma$. The modulus of $\Gamma$ is defined as follows: $M(\Gamma)^{\gamma}=\inf _{\rho \in \operatorname{adm} \Gamma} \int_{D} \rho^{n}(x) d m(x)$. Let $Q: D \rightarrow[0, \infty]$ be a Lebesgue measurable function. A mapping $f: D \rightarrow D^{\prime}$ is called a ring $Q$-mapping at a point $x_{0} \in \bar{D}$, if the inequality $\left.M\left(f\left(\Gamma\left(S\left(x_{0}, r_{1}\right), S\left(x_{0}, r_{2}\right), A\left(x_{0}, r_{1}, r_{2}\right)\right)\right)\right) \leqslant \int_{A\left(x_{0}, r_{1}, r_{2}\right) \cap D} Q(x) \cdot \eta^{n}\left(\left|x-x_{0}\right|\right)\right) d m(x)$ holds for some $r_{0}=r_{0}\left(x_{0}\right)>0$, for all $0<r_{1}<r_{2}<r_{0}$ and for any measurable function $\eta:\left(r_{1}, r_{2}\right) \rightarrow[0, \infty]$ with $\int_{r_{1}}^{r_{2}} \eta(r) d r \geqslant 1$. A mapping $f$ of $D$ onto $D^{\prime}$ is called closed if $C(f, \partial D) \subset \partial D^{\prime}$, where, as usually, $C(f, \partial D)$ is a limit set of $f$ on $\partial D$. Let $h(x, y)$ denotes the chordal distance between points $x, y \in \overline{\mathbb{R}^{n}}$, let $h(A, B)$ denotes the chordal distance between the sets $A, B \subset \overline{\mathbb{R}^{n}}$, and let $h(E)$ be the chordal diameter of the set $E$ in $\overline{\mathbb{R}^{n}}$. For $E \subset \overline{\mathbb{R}^{n}}$ and $\delta>0$, denote by $\Re_{Q, \delta, E}(D)$ the family of all open discrete closed ring $Q$-mappings $f: D \rightarrow \overline{\mathbb{R}^{n}} \backslash E$ in $\bar{D}$ with following condition: for every domain $D_{f}^{\prime}=f(D)$ there is a continuum $K_{f} \subset D_{f}^{\prime}$ such that $h\left(K_{f}\right) \geqslant \delta$ and $h\left(f^{-1}\left(K_{f}\right), \partial D\right) \geqslant \delta>0$. Let $q_{x_{0}}(r):=\frac{1}{\omega_{n-1} r^{n-1}} \int_{\left|x-x_{0}\right|=r} Q(x) d S$, where $d S$ is an area element, $q_{b}^{\prime}(r):=\frac{1}{\omega_{n-1} r^{n-1}} \int_{|x-b|=r} Q^{\prime}(x) d S$ and $Q^{\prime}(x)=\max \{Q(x), 1\}$.

Theorem. Suppose $D$ is locally connected on $\partial D$, and $D_{f}^{\prime}=f(D)$ are uniformly equicontinuous for all $f \in \Re_{Q, \delta, E}(D)$. Let $E$ be a set of positive capacity. Suppose that one of the following conditions holds: 1) either $Q \in F M O$ in $\bar{D}$ or 2) $\int_{0}^{\beta\left(x_{0}\right)} \frac{d t}{t q_{x_{0}}^{\prime} \frac{1}{n-1}(t)}=\infty$ for some $\beta\left(x_{0}\right)>0$ at every point $x_{0} \in \bar{D}$. Then every $f \in \Re_{Q, \delta, E}(D)$ has a continuous extension to $\bar{D}$ and the family $\Re_{Q, \delta, E}(\bar{D})$ consisting of all extended mappings $\bar{f}: \bar{D} \rightarrow \overline{\mathbb{R}^{n}}$ is equicontinuous in $\bar{D}$.

Example 1. The family $f_{n}(z)=z^{n}$ of the unit disc onto itself, $n=1,2, \ldots$, is an example of equicontinuous family of mappings in $D$, what is not so on $\partial D$. The reason is violation of conditions $h\left(K_{f}\right) \geqslant \delta$ and $h\left(f^{-1}\left(K_{f}\right), \partial D\right) \geqslant \delta>0$ in the definition of the class $\Re_{Q, \delta, E}(\mathbb{D})$ and in conditions of the theorem. Worth noting that this family consist of ring 1-mappings.

Example 2. To obtain a similar "good" family of mappings, we put $f_{n}(z)=\left(\frac{z+\frac{1}{n}}{1+\frac{z}{n}}\right)^{2}, n \in$ $\mathbb{N} \backslash\{1\}$. The mappings $f_{n}$ are open, discrete, closed and at the same time they are 1-mappings. If we put $A=\left[0, \frac{1}{2}\right]$, then $f_{n}(A)=\left[\frac{1}{n^{2}},\left(\frac{n+2}{2 n+1}\right)^{2}\right]$. Then in definition of class $\Re_{Q, \delta, E}(\mathbb{D})$ we put $Q \equiv 1, E=\mathbb{C} \backslash \mathbb{D}, K_{f_{n}}=f_{n}(A)$ and $\delta=\frac{1}{10}$. Now, $f_{n} \in \Re_{1,1 / 10, \mathbb{C} \backslash \mathbb{D}}(\mathbb{D})$ for large enough $n \in \mathbb{N}$. 